University of New Hampshire

University of New Hampshire Scholars' Repository

2-1993

\title{
Calculation of acoustic parameters by a filter-correlation method
}

\author{
Robert C. Courtney \\ Geological Survey of Canada \\ Larry A. Mayer \\ University of New Hampshire, larry.mayer@unh.edu
}

Follow this and additional works at: https://scholars.unh.edu/ccom_affil

Part of the Geology Commons, Oceanography and Atmospheric Sciences and Meteorology Commons, and the Sedimentology Commons

\section{Recommended Citation}

The following article appeared in: R. C. Courtney and L. A. Mayer, 'Calculation of acoustic parameters by a filter-correlation method', The Journal of the Acoustical Society of America, vol. 93, no. 2, p. 1145, 1993. and may be found at http://dx.doi.org/10.1121/1.405509.

This Article is brought to you for free and open access by the Center for Coastal and Ocean Mapping at University of New Hampshire Scholars' Repository. It has been accepted for inclusion in Affiliate Scholarship by an authorized administrator of University of New Hampshire Scholars' Repository. For more information, please contact Scholarly.Communication@unh.edu. 


\title{
Calculation of acoustic parameters by a filter-correlation method
}

\author{
Robert C. Courtney \\ Geological Survey of Canada, Atlantic Geoscience Centre, Dartmouth, Nova Scotia B2Y 4A2, Canada \\ Larry A. Mayer \\ Ocean Mapping Group, Department of Survey Engineering, University of New Brunswick, Fredericton, New \\ Brunswick E3B 5A3, Canada
}

(Received 24 September 1990; revised 17 September 1992; accepted 25 September 1992)

\begin{abstract}
This paper presents the filter correlation method, a technique for extracting consistent and accurate estimates of attenuation parameters from acoustic waveform data. The method minimizes problems associated with short time windows and multipath secondary arrivals. The method comprises two stages: a causal passband filter stage followed by a cross-correlation step. The results of the filter-correlation estimator are compared to those of the spectral difference approach for short time series with and without a secondary multipath arrival. Preliminary analyses of acoustic data collected on cored marine silts and clays show the attenuation properties of these materials cannot be described by a constant $Q$ mechanism. The filter correlation method refines estimates of frequency-dependent velocity, revealing a small but systematic anisotropy between measurements made parallel and transverse to the sediments' bedding plane. The observed velocity anisotropy can be modeled by assuming layered porosity variations in the cored sediments. No systematic anisotropy in attenuation was observed.
\end{abstract}

PACS numbers: 43.85.Dj, 43.40.Ph, 43.85. - e

\section{INTRODUCTION}

The characterization of materials by acoustic methods is widely used in many fields of science. Typical acoustic measurements of a medium include compressional and shear wave velocity and attenuation; the manner in which these properties vary with frequency can yield useful information on the nature of the target medium. For example, the $P$-wave attenuation of seismic waves in marine sediments (e.g., Schock et al. ${ }^{1}$ ) can be related to the porosity, grain size, and interstitial fluid of the sediment matrix. ${ }^{2-11}$ In medical applications, estimates of ultrasonic attenuation have been used to characterize soft tissues. ${ }^{12-14}$

This paper describes a method for the calculation of frequency-dependent compressional wave velocity and attenuation parameters derived from transmission waveform data. The method is robust for estimates based on small window sizes in which Fourier methods cannot resolve spectral detail; it also gives superior estimates in the presence of secondary multipath arrivals. It is a two-stage method comprising a causal passband filter followed by a cross-correlation step. A comparison using synthetic data of the filter-correlation method to the spectral difference method is given for variable window lengths and secondary arrivals.

This method has been developed to analyze ultrasonic waveform data collected in split sediment piston cores. Pairs of ultrasonic transducers are inserted directly into split cores and waveform data are collected to ascertain $P$-wave velocity and attenuation characteristics of the sediment. Heuristic examples given in this paper directly reflect this application, although the method could equally be applied to a variety of other waveform data (e.g., earthquake seismic coda, VSP data, etc.). Preliminary attenuation results from cores in high-porosity marine clays are discussed.

\section{BACKGROUND}

The amplitude $A$ of a monochromatic acoustic wave decays exponentially with distance $\delta x$ as it moves through a medium:

$$
A(f, \delta x)=A_{0} e^{-\alpha(f) \delta x}
$$

The attenuation $\alpha(f)$ does not necessarily vary linearly with frequency $f$. A power-law parametrization of $\alpha(f)$ is given by

$$
\alpha(f)=k f^{N},
$$

where $k$ is the attenuation coefficient and $N$ is the power-law exponent. Also, $N$ may vary with frequency; for instance, the Biot model ${ }^{2-11,15}$ for saturated marine sediments predicts that $N$ may vary from 2 to $1 / 2$ as the frequency of the wave increases from the seismic to the ultrasonic wave bands. Rayleigh scattering ${ }^{16}$ is characterized by $N$ equal 4 .

If the material exhibits a linear dependence on frequency $(N=1)$ then it is termed a constant $Q$ material. In this case, $Q$ and $\alpha$ are related by

$$
Q=\pi f / \alpha v,
$$

where $v$ is the acoustic velocity. However, any real material cannot be characterized by $N=1$ over all frequencies as such a material would exhibit a noncausal acoustic response. $^{17}$

Most recent studies ${ }^{18-20}$ on transmission or reflection waveforms have used Fourier transform techniques to estimate the frequency dependence of attenuation. In this ap- 

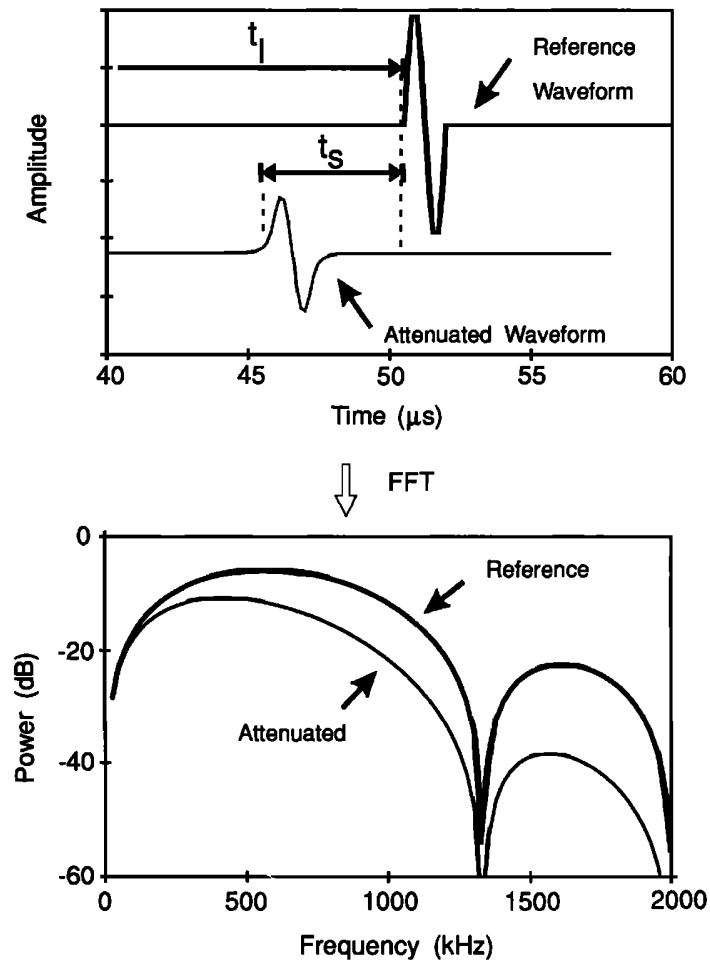

FIG. 1. Two waveforms, with and without effects of attenuation, are shown in the upper plot. The reference waveform arrives at time $t_{1}$, while the attenuated waveform arrives a time interval $t_{s}$, earlier indicative of higher velocities in the dissipative media. The unattenuated waveform is taken as the reference waveform. The spectra of the two are computed and compared in lower frame. The relative rolloff of the attenuated waveforms is used to calculate the attenuation in the spectral ratio method.

proach, an attenuated waveform is windowed and the time series is transformed into the spectral domain with an FFT (Fig. 1). An estimate of an unattenuated waveform is obtained by calibrating transmitter-receiver pairs in a nondissipative medium, or by assuming that the source characteristics are known.

Let $\hat{W}_{c}\left(f_{n}\right)$ be a magnitude of the amplitude spectrum of an unattenuated waveform (the reference waveform) at each discrete frequency $f_{n}$ of the transform, and let $\hat{W}\left(f_{n}\right)$ be the estimate of the attenuated waveform. If the waveforms have traversed a distance $\delta x$, then the estimate of attenuation $\alpha(f)$ is given by

$$
\alpha\left(f_{n}\right)=\frac{-\ln \left[\hat{W}\left(f_{n}\right) / \hat{W}_{c}\left(f_{n}\right)\right]}{\delta x}
$$

or

$$
\alpha\left(f_{n}\right)=\frac{\ln \left[\hat{W}_{c}\left(x_{n}\right)\right]-\ln \left[\hat{W}\left(f_{n}\right)\right]}{\delta x} .
$$

A least-squares line is fit to values of $\log (\alpha)$ plotted against frequency; the values of $k$ and $N$ are derived from the intercept and slope of the fit, respectively. This technique is called the spectral difference or the spectral ratio method. ${ }^{20,21}$

Although this approach is simple and straightforward, difficulties arise when multiple arrivals of the transmitted energy fall within the selected sampling window. This effect has been shown to be a significant and sometimes dominant effect on estimates of attenuation derived from seismic reflection data ${ }^{22-24}$ Unfortunately, single arrivals cannot always be separated or isolated; bandlimited energy necessarily arrives in wave packets of finite length that may overlap following events. The frequency content of the waveform (or, equally, the wave packet width) determines the minimum duration of the sampling window if one wishes to resolve spectral peaks and reduce effects of spectral sidelobe leakage. ${ }^{25}$ If a window brackets less than several cycles of the dominant frequency in the waveform, then Fourier transform estimates of spectral energy are less accurate. Thus both the window size and presence of secondary arrivals place strong constraints on the applicability of the FFT spectral difference method. These effects are illustrated in Sec. III.

\section{FILTER-CORRELATION METHOD}

The filter-correlation method is a two-stage procedure that can give superior estimates of attenuation when length of time windows must be reduced to minimize the effects of secondary multipath arrivals. Assume that two waveforms are available for analysis, an unattenuated reference waveform $w^{c}(t)$ and an attenuated waveform $w(t)$, where $t$ is time (Fig. 2). The waveforms have been sampled $N$ times at equal intervals $\delta t$, in a window of length $T=N \delta t$. The value of $w(t)$ at a specific time $k \delta t$ is denoted by $w_{k} ; k=1, N$.

The waveforms in Fig. 2 were derived from measured signals collected in the lab. The reference waveform was taken from a digitized signal transmitted between a pair of piezoelectric transducers inserted in distilled water. The attenuated waveform was generated by applying a pseudoattenuation filter [Eqs. (1) and (2)] to the reference. The waveforms contain two spectral power peaks near 200 and $800 \mathrm{kHz}$, corresponding to longitudinal and radial resonant oscillations of the crystal transducers. The data were sampled at a rate of $20 \mathrm{MHz}$ and most of the energy in the waveform lies between 100 and $1200 \mathrm{kHz}$. The oversampling in time relative to the Nyquist criterion allows an accurate temporal resolution in the following correlation step. This

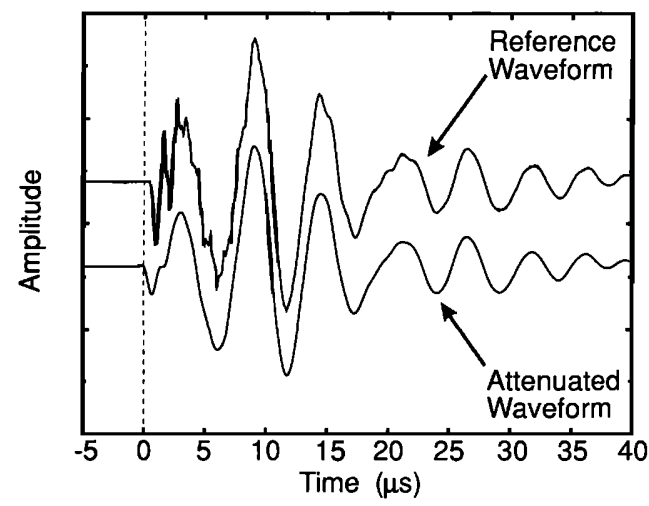

FIG. 2. The test reference waveform and the filtered attenuated waveform are plotted for an attenuation of $k=2.0 \times 10^{-3}$ and $N=1.7$ giving an attenuation $\alpha=252 \mathrm{~dB} / \mathrm{m}$ at $1000 \mathrm{kHz}$. The attenuated pulse was assumed to have traveled $\delta x=0.07 \mathrm{~m}$. 
temporal resolution ultimately determines the resolution of velocity dispersion.

The first arrival of energy in the reference waveform occurs at time $t_{1}$ (Fig. 1); in practice the position of first arrival is determined with a simple threshold criterion. The attenuated waveform has traveled a distance $\delta x$ through a dissipative media. The first arrival of energy in the attenuated waveform is aligned with that of the reference waveform by applying a time shift $t_{s}$. This time shift gives the preliminary estimate of the velocity of the media, $v_{0}=\delta x /\left(t_{1}-t_{s}\right)$. Both reference and attenuated waveforms may contain secondary reflections from the boundaries of the measurement apparatus. A subjective choice is made to choose a window that excludes these secondary arrivals. Other criteria for choosing the window size will be discussed in subsequent sections.

The first step of the method applies a series of causal bandpass filters to the entire recorded length of the reference and attenuated waveforms. A causal filter is used to prevent the migration of energy of secondary arrivals backward in the filtered time series. Noncausal filters, such as a zero phase filter, would allow contaminant energy to leak back into windows used for waveform comparison.

This procedure is applied for a number of discrete passbands for which significant energy is present in the waveform; the number of frequency bands, $n$ band, is usually chosen to range from four to eight. The choice of the number of passbands and the width of each passband is somewhat arbitrary and is based on the characteristics of the waveforms under study. The filtered estimate of the attenuated data $w^{j}$ in passband $j$ is given by

$$
W_{k}^{j}=\sum_{m=1}^{M} g_{m}^{j} W_{(m-k)},
$$

where $g_{m}^{j}$ are the passband filter coefficients of total length $M$. The filtered calibration data $W^{c j}$ are generated in the same way.

In this paper, an eight-pole causal Butterworth filter routine modified from Kanasewich ${ }^{26}$ was used to generate the filter coefficients. This filter has a dropoff of $3 \mathrm{~dB}$ at the bandpass limits and a rolloff of $48 \mathrm{~dB} /$ decade. Other causal filters could be used in this stage although no comparison between filter types has been made by the authors.

The result of the first stage of the process is depicted in Fig. 3 for six passbands ranging from $300-1100 \mathrm{kHz}$. The relative decrease of the amplitude of the attenuated data with respect to the calibration data shows an increasing attenuation with frequency. The relative offset of the peaks of the attenuated waveform reveals frequency-dependent velocity differences between the calibration and the attenuated data in each separate waveband. The change in this offset with frequency is a measure of the velocity dispersion of the wave.

The second stage of the process entails a cross correlation between the reference and attenuated waveforms in each of the passbands within the limits of a window of the filtered time series. No taper is applied to the data, other than the choice of a prearrival and post-arrival time. The filtered attenuated signal is shifted back and forth to align
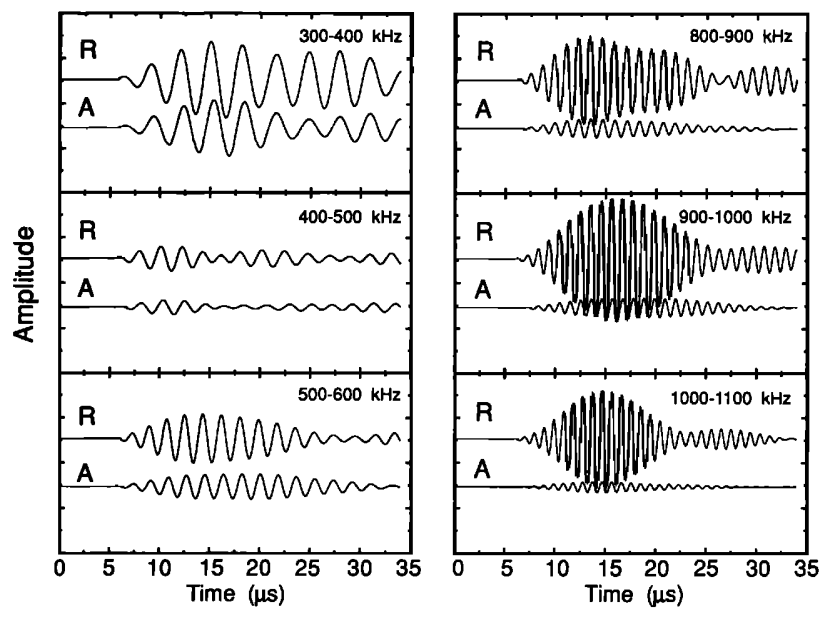

FIG. 3. The waveform data in six passbands are plotted after the first stage of the method. The amplitudes of the attenuated waveforms (A) are plotted relative to the reference $(R)$. The progressive decay in relative amplitude with frequency is obvious.

peaks with the reference by up to a maximum of one halfcycle of the mean passband frequency. The cross correlation, if continued outside this limit, would exhibit multiple local maxima offset by the period of the mean frequency of the passband. If the initial alignment of the reference and attenuated waveforms is in error by more than this limit, then errors in choosing the correct local maxima for calculation of the time shift will arise. The cross correlation reaches a maximum at a time lag, $t_{d}^{j}=d^{j} \delta t$, when the peaks in the attenuated waveform align with the peaks in the reference waveform; this delay is caused by relative dispersion or velocity differences between the reference and the attenuated waveform. The maximum cross correlation $R^{j}$, for a time lag $t_{d}^{j}$, in passband $j$ is given by

$$
R^{j}=\frac{1}{N} \sum_{k=1}^{N} w_{(k+d)}^{j} w_{k}^{c j}\left(\left|w^{j}\right|\left|w^{c j}\right|\right)^{-1}
$$

where $\left|w^{j}\right|$ is the root-mean energy in the attenuated trace

$$
\left|w^{j}\right|=\sqrt{\frac{1}{N} \sum_{k=1}^{N}\left(w_{k+d}^{j}\right)^{2}}
$$

and $\left|w^{c j}\right|$ is the root-mean energy in the reference trace

$$
\left|w^{c j}\right|=\sqrt{\frac{1}{N} \sum_{k=1}^{N}\left(w_{k}^{c j}\right)^{2}} .
$$

The cross-correlation process is used to align attenuated waveform data with the reference waveform. Once aligned, estimates of amplitude differences caused by attenuation can be easily made.

Estimates of the mean frequency in each passband are calculated by averaging the mean of the zero crossings of the filtered waveforms. If $\delta t_{z}^{j}$ is the mean time between zero crossings in the waveform after the time of first arrival of coherent energy in each passband, then the mean frequency of the attenuated waveform is defined as

$$
f_{m}^{j}=\left(2 \delta t_{z}^{j}\right)^{-1}
$$



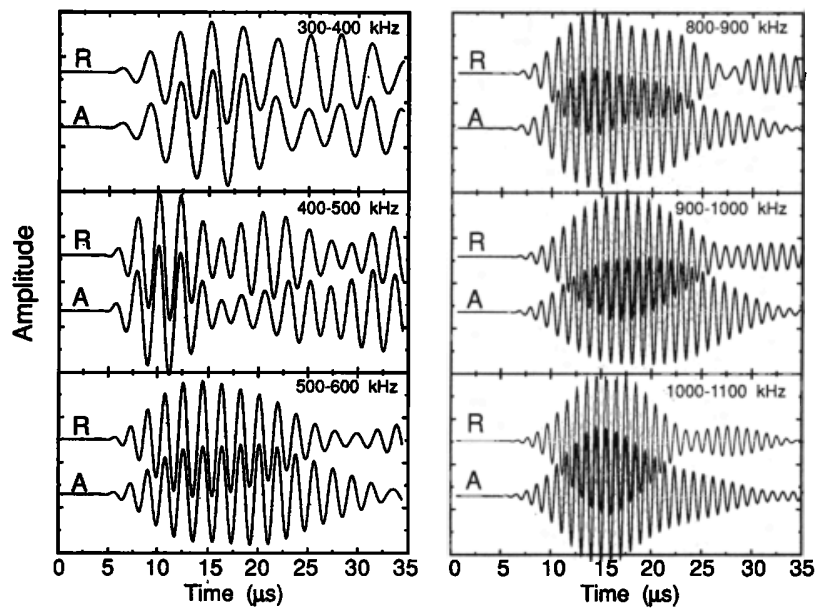

FIG. 4. The waveform data in six passbands are plotted after the crosscorrelation stage. The time shifts and calculated amplitude ratios have been applied to the attenuated waveforms in order to display maximal cross correlation with the reference waveforms. This misfit between the reference and attenuated waveforms is small; the correlation value (see text) exceeds 0.99 for each set.

and similarly $f_{m}^{c j}$ for the reference waveform. Other methods were tested to estimate the mean frequency (e.g., average of the passband limits, maximum entropy analysis, cross-correlation techniques) but the zero crossing method proved most simple, most reliable and most accurate.

In Fig. 4, the reference and attenuated waveforms are plotted in each passband after the correlation stage where relative time shifts and mean energies have been calculated. The waveforms are normalized by their root-mean energy and the attenuated data shifted by $t_{d}^{j}$. This figure shows that the filter-correlation method essentially performs a waveform fitting in each of the passbands, extracting information about time delay and amplitudes in each discrete frequency band.

Estimates of frequency-dependent velocity $v^{j}$ in each pass band are calculated using the time delay offsets and the preliminary time shifts

$$
v^{j}=\frac{\delta x}{t_{l}-t_{s}+t_{d}^{j}} .
$$

Estimates of the attenuation $\alpha^{j}$, in each passband are given by

$$
\alpha^{j}=\frac{-\ln \left(\left|w^{j}\right| /\left|w^{c j}\right|\right)}{\delta x} .
$$

The calculation of $k$ and $N$ in Eq. (2) is made by a leastsquares fit straight line between $\log \left(\alpha^{j}\right)$ against the average between the mean frequencies in the attenuated and reference passbands, as shown in Fig. 5.

\section{COMPARISON WITH THE SPECTRAL METHOD USING SYNTHETIC DATA}

A series of comparisons between the filter-correlation method and the spectral difference method were made on synthetic data shown in Fig. 2. Results for two different sets of trials are reported here. The first series compares results of the two methods as a function of the size of the data window.
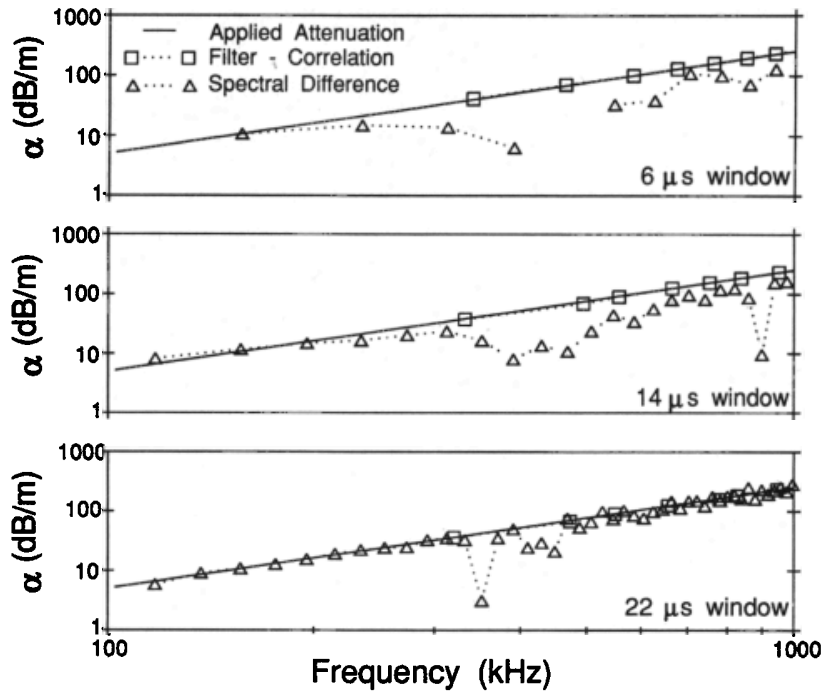

FIG. 5. Attenuation estimates of the filter-correlation method (square symbol) and the spectral ratio method (triangle) are plotted as a function of frequency. The solid straight line is the target attenuation.

The second series contrasts predictions for an attenuated waveform that contains a secondary multiple arrival. These comparisons serve to illustrate the effectiveness of the filtercorrelation method.

In both cases the attenuated wave was filtered with the following attenuation parameters: $k=0.0174 \mathrm{~dB} / \mathrm{m}-\mathrm{kHz}^{N}$ and $N=1.7$. The distance traveled by the attenuated wave $\delta x$ was set to $0.0715 \mathrm{~cm}$. These values correspond to a net attenuation of $252 \mathrm{~dB} / \mathrm{m}$ at $1000 \mathrm{kHz}$. A progressive phase shift was applied to induce a linear increase of velocity by 10 $\mathrm{m} / \mathrm{s}$ from 100 to $1000 \mathrm{kHz}$.

For calculation of FFT estimates, waveforms within the comparison window were multiplied by a Welsh window. ${ }^{25}$ The Welsh window applies the weighting operator,

$$
a_{j}=1-\left(\frac{j-\frac{1}{2}\left(N_{s}-1\right)^{2}}{\frac{1}{2}\left(N_{s}+1\right)}\right) ; j=1, N_{s},
$$

over the length of the window $N_{s}$. The choice of the window type affects the results of the spectral difference method, underlining the inherent difficulties with this approach.

FFT spectral estimates between 100 to $350 \mathrm{kHz}$ and 650 to $1000 \mathrm{kHz}$ were used to compute attenuation parameters in the spectral difference method, avoiding a notch in the power spectra at $500 \mathrm{kHz}$. Eight passbands were used in the filter-correlation method: 300 to $400 \mathrm{kHz}, 400$ to $500 \mathrm{kHz}$, 500 to $600 \mathrm{kHz}, 600$ to $700 \mathrm{kHz}, 700$ to $800 \mathrm{kHz}, 800$ to 900 $\mathrm{kHz}, 900$ to $1000 \mathrm{kHz}$, and 1000 to $1100 \mathrm{kHz}$.

In the first test series effects of window length are tested. The comparison window was chosen with a fixed pre-arrival interval of $4 \mu \mathrm{s}$ with a variable period of 5 to $25 \mu \mathrm{s}$ after the first break of coherent energy. Estimates of attenuation versus frequency were generated and estimates of $k$ and $N$ were derived from a least-squares fit to the data. The results are plotted in Fig. 6; estimates of $\alpha$ at $1000 \mathrm{kHz}$ and $N$ are given as a function of the the sum of the pre- and post-arrival window lengths. 

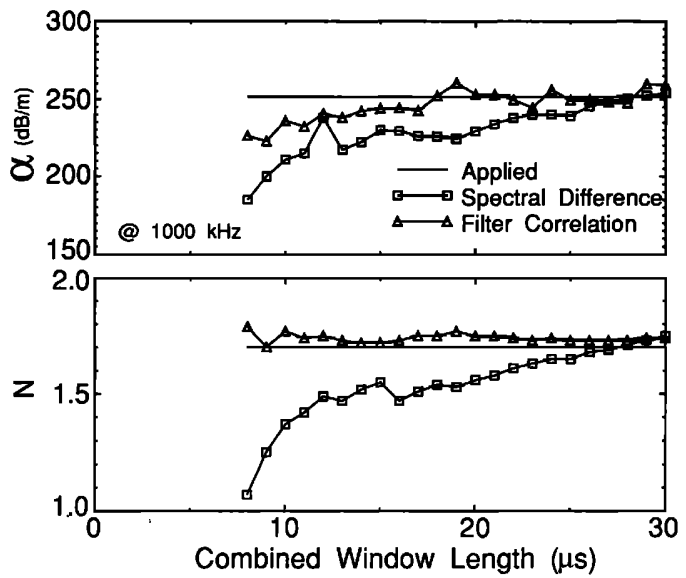

FIG. 6. The estimates of $\alpha$ and $N$ at $1000 \mathrm{kHz}$ are plotted as a function of the post-arrival window size. The filter-correlation estimates are marked by the triangle symbol, while the spectral difference (FFT) estimates are marked by the square symbols.

The filter-correlation method produces superior estimates of $\alpha$ and $N$. The spectral difference method seriously underestimates $\alpha$ for window sizes less than $18 \mu \mathrm{s}$; errors approach $50 \%$ of the target value in the worst case. At least $25 \mu$ s of uncorrupted data are needed to make good estimates of the attenuation parameters using the FFT method, while a window of 15 suffices for the filter-correlation method. FFTderived estimates of $N$ show considerable error and no confidence can be placed in results produced by this method.

A second series of tests were made with a secondary multiple that arrives $10 \mu \mathrm{s}$ after the first arrival of coherent energy. The amplitude of the multiple was set to -0.5 times that of the attenuated wave in Fig. 2 . The multiple waveform and the reference waveform are shown in Fig. 7. The effects of the secondary multiple are seen in the later part of the waveforms.

The predictions of $\alpha$ and $N$ for the second test series are depicted in Fig. 8. The filter correlation method continues to make better estimates of $\alpha$ until $20 \mu \mathrm{s}$, well after the arrival of the secondary multiple. The estimate of $N$ progressively but uniformly degrades, erring by $-8 \%$ at $22 \mu \mathrm{s}$. The spectral difference method fails to make a comparable estimate of these acoustic parameters and at no time produces acceptable results. By $23 \mu \mathrm{s}$, the estimate of $N$ is $25 \%$ below that applied.

These tests of synthetic data confirm the stability and accuracy of the filter-correlation method using short window lengths in the presence of a secondary multiple. Although the multiple problem is not completely removed, the effects of the multiple on the results predicted by the filtercorrelation method are greatly reduced. The spectral difference method results in more variable estimates of the acoustic parameters as the length of the time window changes; a meaningful value based on these estimates would be hard to constrain.

\section{APPLICATION TO FIELD DATA}

In the spring of 1987 , a set of compressional wave data was collected on large diameter $(11.4 \mathrm{~cm})$ piston cores taken

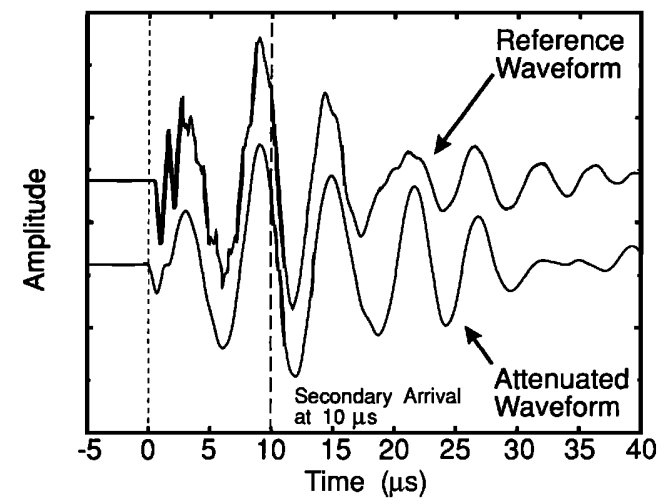

FIG. 7. The attenuated waveform has been augmented by a secondary arrival at $10 \mu$ s after the time of the first arrival of energy. The secondary arrival has an amplitude of -0.5 times the attenuated waveform in Fig. 2.

from Emerald Basin, a Quaternary deposit of unconsolidated glaciomarine clay and silts located approximately $100 \mathrm{~km}$ south-southeast of Halifax, Nova Scotia. ${ }^{27}$ Piston cores were split normally within $24 \mathrm{~h}$ of collection, and acoustic properties were measured immediately thereafter. Biogenic gas caused expansion and cracking of some of the cores. Acoustic measurements made on these cores showed a significant reduction in the amplitude of transmitted sound and these measurements were discarded. In situ measurements ${ }^{28}$ might avoid this problem; however, velocity measurements at more than one direction to the bedding plane would be difficult with a remotely deployed device. A more complete discussion of the data collection and analysis of these data are the subject of another paper; ${ }^{29}$ only a sample of waveform data from core 87003-004 is discussed here.

Compressional wave data were collected using a marine sediment acoustic measurement system as described by Baldwin. ${ }^{30}$ The system was modified by the authors, incorporating digital recording, automatic first break detection, and processing of the acoustic waveform data. The system uses two pairs of piezoelectric transducers of small diameter

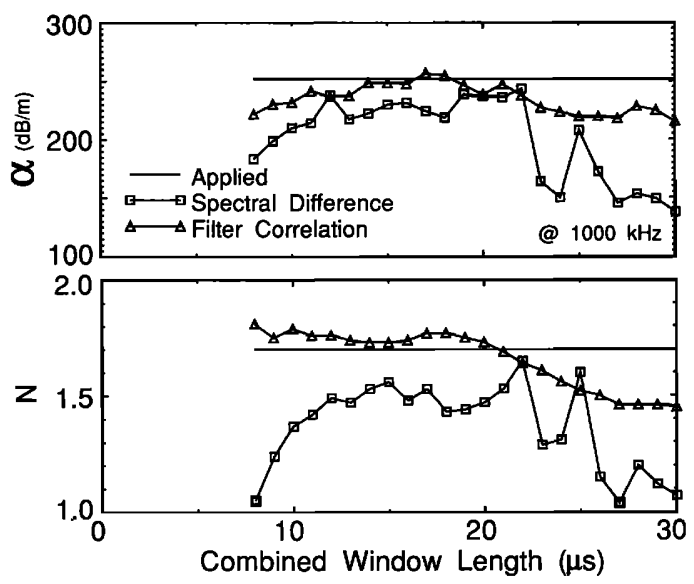

FIG. 8. Estimates of $\alpha$ and $N$ at $1000 \mathrm{kHz}$ as a function of window size for the case of the secondary multiple given in Fig. 7. The spectral difference (FFT) estimates are highly sensitive to the presence of the multiple. 
$(0.635 \mathrm{~cm})$. Each transducer has two major electromechanical resonances near 200 and $800 \mathrm{kHz}$. The transducers are potted in aluminum probes that can be inserted directly into the split piston core with minimal disturbance of the sediment. Each pair of probes forms a source and receiver combination. A high-voltage, short-duration $(2 \mu \mathrm{s})$ pulse is applied to the transmitter and the time of flight of the transmitted energy to the receiver is used to estimate the sediment velocity. The received waveform is digitized and stored on disk.

The geometry of the two set of probes is rigidly fixed. One set of probes is aligned along the axis of the piston core and has a transducer separation of $0.07 \mathrm{~m}$. The other pair is aligned transverse to the core axis and has a separation of $0.045 \mathrm{~m}$. The separation between the transmitter and receiver pair is calibrated in distilled water. The temperature of the distilled water and the sediment is continuously recorded during the measurement. The waveform passed through the distilled water is used as the reference waveform, making corrections for waveform geometrical spreading unnecessary. In these measurements, sediment velocities and attenuation values are computed relative to that of distilled water, a known standard.

\section{Time $(\mu s)$}
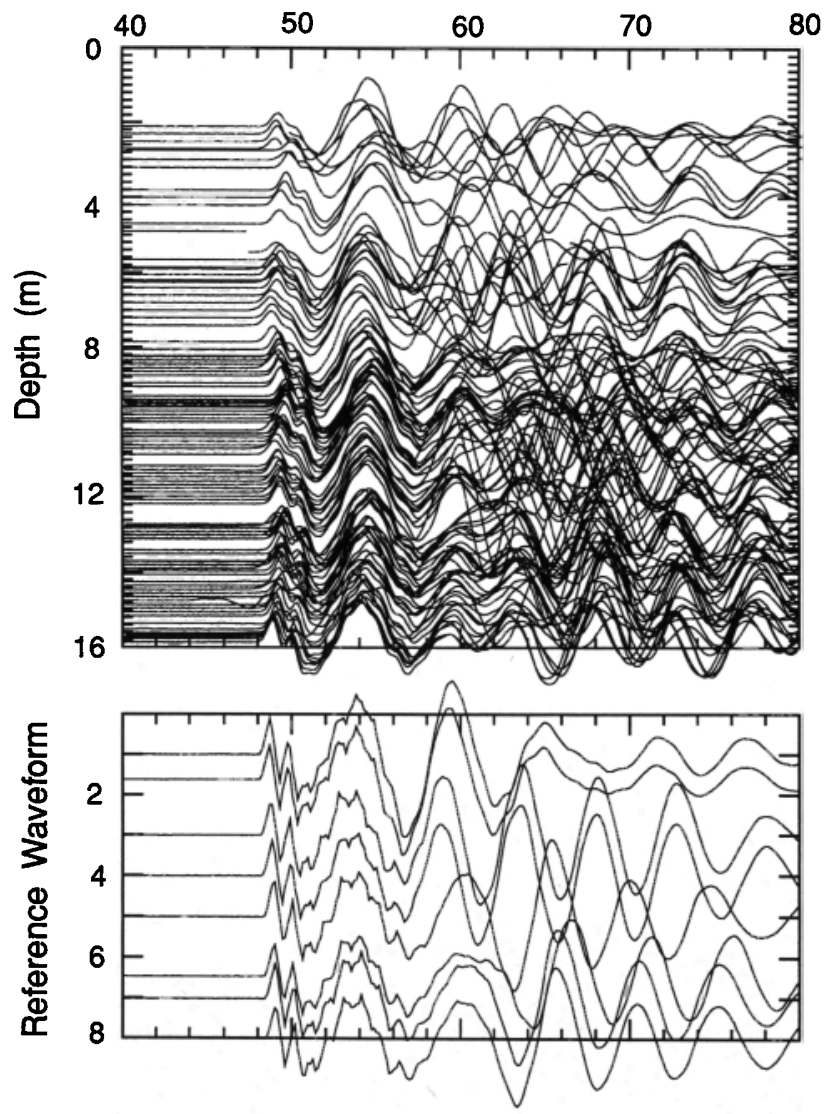

FIG. 9. The top diagram shows waveform data taken through clay/slit sediments from Emerald Basin, while the lower diagram shows calibration waveforms collected in distilled water. Both data sets show coherency in the first $10 \mu$ s between adjacent waveforms after the first arrival of energy. The arrival of secondary reflections and other multiples mask coherency later on in the time series.
Preliminary estimates of the first arrival of energy are made automatically using a simple amplitude threshold criterion; the operator has the option to manually reposition the first break position, although it was not necessary with these data. Measurements of velocity were made every 10 to $20 \mathrm{~cm}$ down the core, over a distance of up to $20 \mathrm{~m}$.

Waveform data for the longitudinal measurements are illustrated in Fig. 9, with with eight waveforms of water calibrations. A visual inspection indicates that the waveforms are highly correlated for the first $10 \mu$ s after the first arrival of energy. After that time, a train of uncorrelated phases arrive at random times.

These secondary arrivals arise from reflection off the top, side, and end boundaries of the split core. The amplitude and arrival time of these multipath arrivals depend critically on the precise position of the transducer pair relative to the boundaries of the sediment sample. In practice, small differences of the transducer positions between successive measurements cannot be avoided. In some cases, the thickness of the split core varies; in other cases, measurements are made near the ends of core sections. In a spectral difference method at least $25 \mu$ s of the waveform would have to be windowed to resolve the parameters with any confidence, which would include significant multipath energy. The filter correlation method however allows the accurate calculation of the attenuation parameters within the physical constraints of the data set.

Emerald Basin core data were processed with eight passbands (those listed in the previous section) and with a pre-arrival length of $4 \mu \mathrm{s}$ and a post-arrival length of $10 \mu \mathrm{s}$. Estimates of velocity $\alpha$ and $N$ at $1000 \mathrm{kHz}$ are plotted in Fig. 10 for both longitudinal and transverse measurements. Since the measurement along each direction is independent, in the absence of significant anisotropy, the results are indicative of the accuracy and repeatability of the method. At each depth in Fig. 10, the extreme excursions of the error bars indicate the longitudinal and transverse measurements, while the

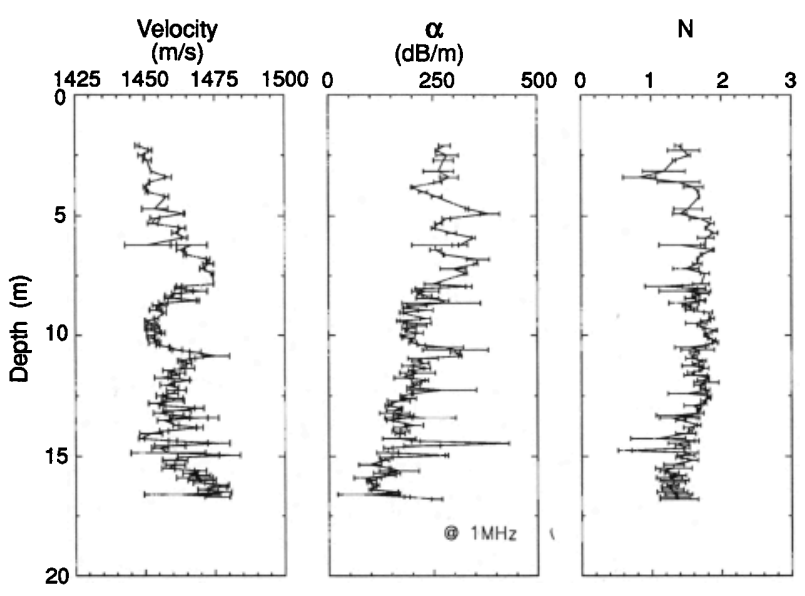

FIG. 10. Estimates of velocity $\alpha$ and $N$ at $1000 \mathrm{kHz}$ are plotted for core 87003-004. The solid line plots the mean between the transverse and longitudinal measurements and the error bars mark excursion of the longitudinal and transverse measurements from the mean. 
center of the error bar plots the average value. The values of velocity have been corrected to a temperature of $10^{\circ} \mathrm{C}$.

The values of velocity are most highly constrained. The transverse and longitudinal measurements generally agree within $5 \mathrm{~m} / \mathrm{s}$. The estimates of attenuation show more relative scatter, but the most agree within $10 \%$ at $1000 \mathrm{kHz}$. The estimates of $N$ average around 1.5 and show an average difference of less than 0.15 . These sediments thus show a markedly nonconstant $Q$ and have high values of $N$ that are in good agreement with those predicted by Biot-Stoll theory. ${ }^{31}$

The passband estimates of velocity at $1000 \mathrm{kHz}$ were uniformly lower than the preliminary estimates based on the first arrival of energy. The average difference between the velocity estimate for the lowest band (300 to $400 \mathrm{kHz}$ ) and the time of flight estimate was $-12.6 \pm 2.5 \mathrm{~m} / \mathrm{s}$. The average difference between the velocity in the lowest passband ( 300 to $400 \mathrm{kHz}$ ) and the highest passband (1000 to 1100 $\mathrm{kHz}$ ) was $-2.9 \pm 1.4 \mathrm{~m} / \mathrm{s}$, weakly resolved in this analysis. The smallest difference between velocity estimates is 1.5 $\mathrm{m} / \mathrm{s}$, determined by the transducer separation and digitization rate. These data indicate that, although dispersion is not important between 300 and $1100 \mathrm{kHz}$, the use of first break estimates of the waveform can significantly bias velocity estimates. This effect may be attributed to the arrival of higher frequency energy above the passbands.

Although a detailed discussion of this data set is presented elsewhere, ${ }^{29}$ the acoustic data present new and useful information about the cored sediment. The velocity data shows a marked increase at $11-\mathrm{m}$ depth. This change correlates with a pronounced change in the character of highfrequency seismic reflectors, and marks a transition from the proglacial Emerald Silt unit to the transgressive LaHave Clay unit. ${ }^{27}$ The transition was not obvious from a visual inspection of the split core.

\section{ANISOTROPY}

$X$-ray photographs of these sediments reveal microscale banding, interpreted as thin layers of sand intercalcated with

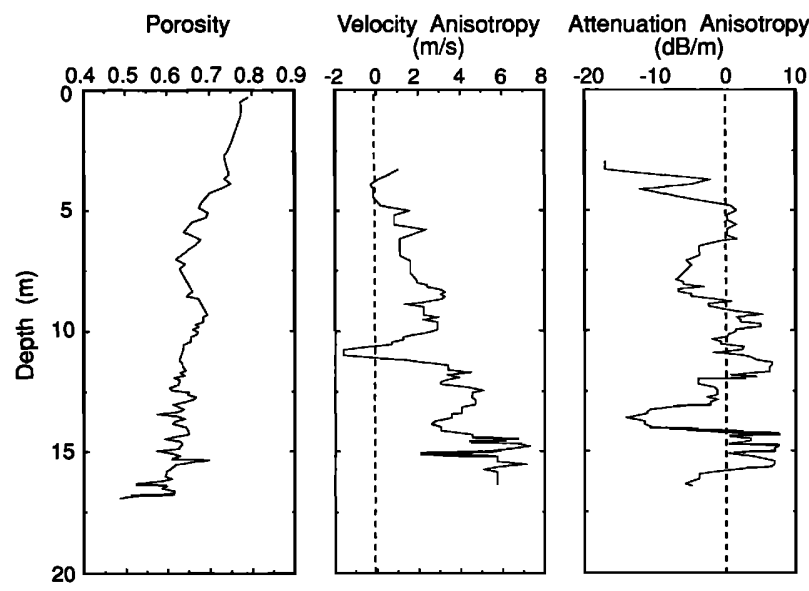

FIG. 11. Porosity is plotted against depth in the core. The anisotropy (the difference between transverse and longitudinal measurements) in both velocity and anisotropy is plotted in the center and right-hand frames. A fivepoint median filter was applied to both anistropy plots before plotting to reduce scatter, highlighting changing trends in these measurements. clay and silt on a subcentimetre scale. It has been predict$\mathrm{ed}^{32,33}$ that a finely layered media should show compressional wave anisotropy, induced by shear coupling between adjacent layers.

In Fig. 11 the velocity anisotropy, expressed here as the difference between the transverse and longitudinal velocities, is plotted against depth in the core. Porosity, derived from bulk density measurements, and the anisotropy in attenuation in the core are also plotted. The velocity anisotropy increases progressively with depth toward a value of 6 $\mathrm{m} / \mathrm{s}$ at $16 \mathrm{~m}$, punctuated by a significant negative excursion of $-2 \mathrm{~m} / \mathrm{s}$ near $11 \mathrm{~m}$. The negative excursion correlates with a sharp increase in the mean velocity (Fig. 10). The velocity anisotropy increase appears to correlate inversely with the porosity, although the regression between the two variables has a low correlation value of less than 0.5 . No consistent trend in the attenuation anisotropy is observed, although the transverse attenuation is generally lower than the longitudinal measurement.

We use a simple two-layer model to investigate the observed anisotropy. Following Brekhovskikh, ${ }^{32}$ the compressional wave velocity $c_{z z}$ for waves propagating perpendicular (corresponding to the longitudinal measurement axis) to the layering is given by

$$
c_{z z}^{2}=\frac{2}{\rho\left[1 /\left(\lambda_{1}+2 \mu_{2}\right)+1 /\left(\lambda_{2}+2 \mu_{2}\right)\right]} .
$$

Here $\lambda_{1}, \mu_{1}, \lambda_{2}$, and $\mu_{2}$ are the Lamé elastic constants of two, repeatedly interbedded, thin layers of equal thickness. It is assumed that the wavelength of the acoustic waves is much greater than the layer thicknesses. The mean density of the two layers is $\rho$. The ratio of the compressional velocity $c_{x x}$ of waves propagating parallel to the layers to $c_{z z}$ is

$$
\left(\frac{c_{x x}}{c_{z z}}\right)^{2}=1+\frac{\left(\mu_{2}-\mu_{1}\right)\left[\lambda_{2}+\mu_{2}-\left(\lambda_{1}+\mu_{1}\right)\right]}{\left[1 /\left(\lambda_{1}+2 \mu_{1}\right)+1 /\left(\lambda_{2}+2 \mu_{2}\right)\right]} .
$$

Levin $^{33}$ gives equivalent formulas using compressional and shear velocities, but the relationships using the Lamé constants are simpler and show clearly the effects of shear coupling between the layers. For example, no velocity anisotropy is predicted when the shear moduli of both layers are equal. Under most circumstances, the ratio is greater than, or equal to, 1 for structurally competent sediments, and the transversely measured velocity should exceed the longitudinal measurement. The numerator of the second term of Eq. (14) can be negative, resulting in a velocity ratio less than 1 , when

$$
\left(\mu_{2}-\mu_{1}\right)\left[\lambda_{2}+\mu_{2}-\left(\lambda_{1}+\mu_{1}\right)\right]<0 .
$$

This condition may be met as the Poisson's ratio $\sigma$ of one of the layers approaches that of a fluid ${ }^{33}(\sigma=0.5)$.

Values of the Lamé parameters for the Emerald Basin sediments must be obtained through inference based on empirical data. Hamilton ${ }^{34}$ derived parametrized estimates for the frame modulus of marine sediments from experimental data. His data are referenced to an overburden pressure of 1 $\mathrm{m}$ or less, appropriate for split core data as presented here. Using Hamilton's expression for natural silty clays, the 
shear modulus as a function of porosity $\phi$ is given by

$$
\mu(\phi)=\frac{3(1-2 \sigma) e^{2.7350-4.25075 \phi}}{2(1+\sigma)} 10^{8} \mathrm{~Pa},
$$

where $\sigma$ is set to 0.3 .

The variation of the compressional wave velocity with porosity has been described frequently in the literature; we will use an expression given by Anderson ${ }^{35}$ based on velocities of sediments found in the depth range $0.1-1.5 \mathrm{~km}$ (Table 4 of Anderson ${ }^{35}$ ) corrected to a temperature of $10^{\circ} \mathrm{C}$ :

$$
v_{a}(\phi)=2478-2724 \phi+1816 \phi^{2} .
$$

A least-squares fit of a quadratic through the velocity data (Fig. 10) plotted against porosity (Fig. 11) collected on the Emerald Basin core yields

$$
v_{b}(\phi)=1782-833 \phi+522 \phi^{2} .
$$

This expression applies within the porosity range of the data ( $0.55-0.78)$; however, its validity at higher and lower porosities must be questioned. Figure 12 plots the observed data against the two quadratic forms. Anderson's expression predicts significantly higher increases in velocity outside the observed porosity range.

Estimates of $\lambda$ are derived from estimates of these velocities using estimates for the shear modulus and bulk density:

$$
\lambda=\left[\rho_{w} \phi+(1-\phi) \rho_{m}\right] v^{2}(\phi)-2 \mu(\phi),
$$

where the fluid density $\rho_{w}$ is $1030 \mathrm{~kg} / \mathrm{m}^{3}$. The density of the grains $\rho_{m}$ of the sediments was measured on the cores, giving mean value of $2780 \mathrm{~kg} / \mathrm{m}^{3}$.

Although the porosity has been used here to predict the values of the elastic parameters, other factors, including grain size, grain alignment, grain contact cementation, grain composition, and consolidation history, will have significant effects. Given these caveats, only the porosity parametrization is employed here.

Using these estimates of $\lambda$ and $\mu$, Eqs. (13) and (14) can be used to predict the velocity difference between transverse and longitudinal measurements as a function of poros-



FIG. 12. The upper curve plots the parametrization of velocity by porosity given by Anderson; ${ }^{35}$ the lower curve is the best-fit quadratic to porosity and velocity values (marked by crosses) measured in the Emerald Basin cores. ity. The abscissa in Fig. 13 (a) is the mean porosity of the two layers, that which would be obtained from bulk measurements. The vertical axis measures the porosity in the layers of the model. A vertical line drawn at a specified value of the mean porosity intersects an anisotropy contour of a chosen value twice; the vertical coordinates at these intersections define a pair of layer porosities which would induce an anisotropy of the value of the specified contour. For example, a vertical line drawn at a mean porosity of 0.65 intersects the $5-\mathrm{m} / \mathrm{s}$ contour at layer porosities equal to 0.45 and 0.85 (the mean of the two is, of course, 0.65 ). Contours are restricted to an area of the plot where the layer porosities are greater than 0 and less than 1.

Figure 13(a) shows that above a porosity of 0.75 , the calculated anisotropy does exceed $5 \mathrm{~m} / \mathrm{s}$; the magnitudes of the shear modulus are too small at these high porosities to have much effect. As the mean porosity falls, the shear modulus increases and larger values of anisotropy can be produced. Near a porosity of 0.5 , a wider range of anisotropy values can be produced, up to $80 \mathrm{~m} / \mathrm{s}$. However, for the largest values, a fluid would have to be interbedded with a layer having no porosity, a physically untenable circumstance for surficial sediments. At a porosity of 0.55 , typical of the bottom section of the Emerald Basin core, layer porosities of 0.38 and 0.72 would induce an velocity difference of $6 \mathrm{~m} / \mathrm{s}$, close to that observed. These porosities are not unreasonable for interbedding of clay and sand layers.

The least-squares fit expression [Eq. (18)] predicts less anisotropy, although the general shape and trends in contours are similar. At a mean porosity of 0.65 , layer porosities of 0.47 and 0.83 would induce a velocity difference of $4 \mathrm{~m} / \mathrm{s}$, roughly $1 \mathrm{~m} / \mathrm{s}$ less than predicted with Eq. (17). At a porosity of 0.55 , layer porosities of 0.34 and 0.76 are needed to get a velocity difference of $6 \mathrm{~m} / \mathrm{s}$. Again, these porosities are not unreasonable for interbedding of clay and sand layers. The least-squares fit expression predicts approximately $80 \%$ of the velocity anisotropy predicted by Anderson's expression.

In summary, the two-layer model can produce velocity anisotropy values similar to those observed in the Emerald Basin cores. At the top of the core where porosity approaches 0.80 , the model predicts that anisotropy cannot exceed 2 to $3 \mathrm{~m} / \mathrm{s}$, in agreement with the observations. As the mean porosity decreases with depth in the core, the theory predicts that higher levels of anisotropy can be produced by porosity variations. Layer porosities must vary between $35 \%$ and $75 \%$ at the bottom of the core to fit the observed velocity data, consistent with clay and sand interbedding.

The model cannot predict a negative anisotropy as observed in the core data. Presumably, the parametrization of the Lamé elastic constants fails in this region. Perhaps the cored material had been disturbed by sampling in this region, resulting in weakened and fluidized layers interbedded with competent layers. No systematic trends in attenuation anisotropy is observed. Any attempt to model the attenuation anisotropy data would likely be overly speculative.

\section{CONCLUSIONS}

This paper has presented the filter-correlation method, a means of extracting consistent and accurate estimates of 
(a)

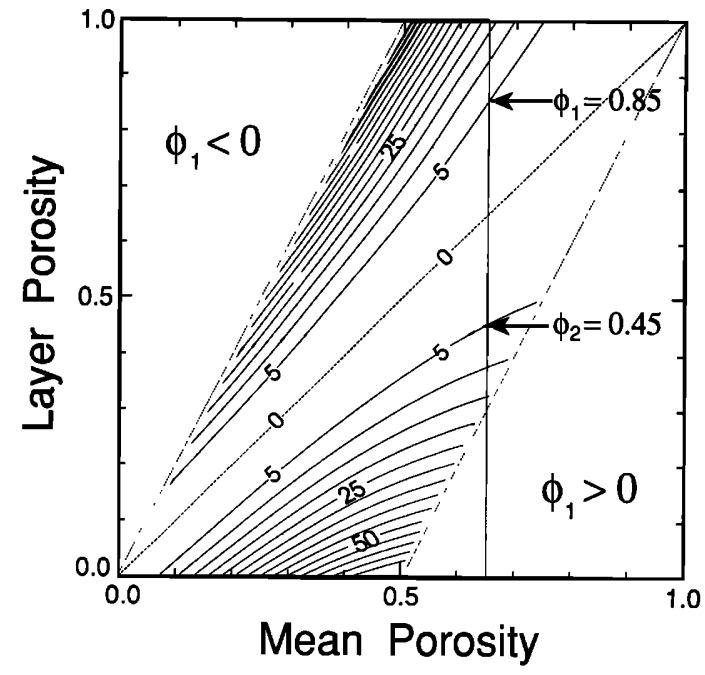

(b)

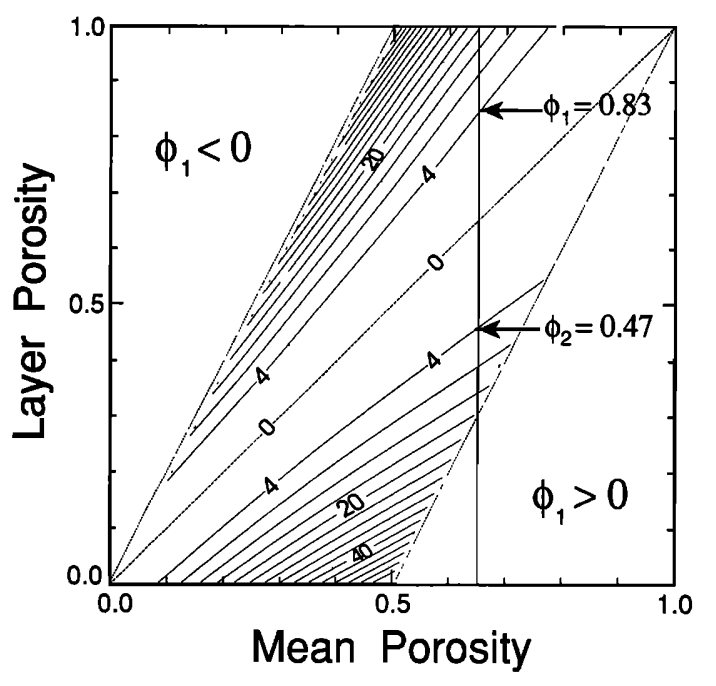

FIG. 13. (a) A contour plot of the difference in velocity for waves propagating parallel and perpendicular to a thinly bedded, two layer model using Anderson's ${ }^{35}$ parametrization of velocity by porosity. The horizontal axis is the mean porosity of the two layers and the vertical axis is the layer porosity. A vertical line at a given value of the mean porosity intersects a contour of a fixed value twice, corresponding to the porosities of each of the two layers. Velocity contours are $5 \mathrm{~m} / \mathrm{s}$. (b) A contour plot of the difference in velocity for waves propagating parallel and perpendicular to a thinly bedded, twolayer model using the least-squares fit quadratic parametrization of velocity by porosity. Velocity contours are $4 \mathrm{~m} / \mathrm{s}$.

acoustic parameters from acoustic waveform data. The method minimizes problems associated with short time windows, which tend to corrupt attenuation estimates made with Fourier spectral techniques. The filter-correlation method makes superior estimates in the presence of a multipath secondary arrival.

Preliminary analyses of acoustic data collected on cored marine silts and clays show that the attenuation properties of these materials cannot be described by a constant $Q$ mechanism. A Biot-Stoll mechanism is more likely. Estimates of velocity dispersion show that dispersion in the range of frequencies between 300 and $1100 \mathrm{kHz}$ is small. However, the arrival of higher frequency data biases the estimate of veloc- ity made by first break detection upward by about $12 \mathrm{~m} / \mathrm{s}$. The filter-correlation method permits the calculation of velocity in a fixed waveband, necessary for accurate modeling of the data.

Accurate estimates are needed to measure compressional wave velocity anisotropy in unconsolidated sediments. The compressional wave velocity parallel to the bedding plane of the sediments is consistently higher than those of the waves propagating perpendicular to the planes, increasing from $0 \mathrm{~m} / \mathrm{s}$ at the surface of the core to $6 \mathrm{~m} / \mathrm{s}$ at 15 $\mathrm{m}$. The error in velocity caused by ignoring this effect is less than $1 \%$. A simple two-layer model suggests that the observed velocity anisotropy is consistent with a decrease in bulk porosity with depth in the core and small-scale interlayering between alternating layers of contrasting porosity.

\section{ACKNOWLEDGMENTS}

The authors would like to acknowledge the support of the U.S. Office of Naval Research (Contract N00014-87-G0114) and the Natural Sciences and Engineering Research Council of Canada. We would like to thank the officers and crew of CSS HUDSON. Kate Moran and Harold Christian provided geotechnical expertise.

'S. H. Schock, L. R. Leblanc, and L. A. Mayer, "Chirp subbottom profiler for quantitative sediment analysis," Geophysics 54, 445-450 (1989).

${ }^{2} \mathrm{M}$. A. Biot, "Theory of propagation of acoustic waves in a fluid-saturated porous solid, I. Low frequency range," J. Acoust. Soc. Am. 28, 168-178 (1956).

${ }^{3} \mathrm{M}$. A. Biot, "Theory of propagation of acoustic waves in a fluid-saturated porous solid, II. Higher frequency range," J. Acoust. Soc. Am. 28, 179191 (1956).

${ }^{4}$ M. A. Biot, "Mechanism of deformation and acoustic propagation in porous media," J. Appl. Phys. 33, 1482-1498 (1962).

${ }^{5} \mathrm{M}$. A. Biot, "Generalized theory of acoustic propagation in porous dissipative media," J. Acoust. Soc. Am. 34, 1254-1264 (1962).

${ }^{6} \mathrm{E}$. L. Hamilton, "Sound attenuation as a function of depth in the sea floor," J. Acoust. Soc. Am. 59, 528-535 (1976).

${ }^{7}$ E. L. Hamilton and R. T. Bachman, "Sound velocity and related properties of marine sediments," J. Acoust. Soc. Am. 72, 1891-1904 (1982).

${ }^{8}$ R. D. Stoll, "Acoustic waves in ocean sediments," Geophysics 42, 715725 (1977).

${ }^{9}$ R. D. Stoll, "Experimental studies of attenuation in sediments," J. Acoust. Soc. Am. 66, 1152-1160 (1979).

${ }^{10}$ R. D. Stoll, "Theoretical aspects of sound transmission in sediments," J. Acoust. Soc. Am. 68, 1341-1350 (1980).

${ }^{11}$ R. D. Stoll, "Marine sediment acoustics," J. Acoust. Soc. Am. 77, 17891799 (1985).

${ }^{12}$ S. A. Goss, R. L. Johnston, and F. Dunn, "Compilation of empirical ultrasonics properties of mammalian tissue II," J. Acoust. Soc. Am. 68, 93108 (1980).

${ }^{13}$ S. Shaffer, D. W. Pettibone, J. F. Havlice, and M. Nassi, "Estimation of the slope of the acoustic attenuation coefficient," Ultrason. Imag. 6, 126138 (1984).

${ }^{14}$ K. A. Dines and A. C. Kac, "Ultrasonic attenuation tomography of soft tissues," Ultrason. Imag. 1, 16-33 (1979).

${ }^{15}$ A. C. Kibblewhite, "Attenuation of sound in marine sediments: A review with emphasis on new low-frequency data,"J. Acoust. Soc. Am. 86, 716737 (1989).

${ }^{16}$ C. S. Clay and H. Medwin, Acoustical Oceanography: Principles and Applications (Wiley-Interscience, New York, 1977), pp. 185-187.

${ }^{17}$ B. J. Brennan and D. E. Smylie, "Linear viscoelasticity and dispersion in seismic wave propagation," Rev. Geophys. Space. Phys. 19, 233-246 (1981). 
${ }^{18}$ G. T. Kuster and M. N. Toksoz, "Velocity and attenuation of seismic waves in two-phase media: Part II. Experimental results," Geophysics 39, 607-618 (1974).

${ }^{19}$ M. Badri and H. M. Mooney, " $Q$ measurements from compressional seismic waves in unconsolidated sediments," Geophysics 52, 772-784 (1987).

${ }^{20} \mathrm{D}$. Goldberg and B. Zinzer, " $P$-wave attenuation measurements from laboratory resonance and sonic waveform data," Geophysics 34, 76-81 (1989).

${ }^{21}$ M. N. Toksoz, D. H. Johnson, and A. Timur, "Attenuation of seismic waves in dry and saturated rocks, I. Laboratory measurements," Geophysics 44, 681-690 (1979).

${ }^{22}$ S. A. Raikes and R. E. White, "Measurements of earth attenuation from downhole and surface seismic recordings," Geophysic. Prospect. 32, 892919 (1984)

${ }^{23}$ J. R. Resnick, “Stratigraphic filtering,” Pageoph 132, 49-65 (1990).

${ }^{24}$ T. W. Spencer, J. R. Sonnad, and T. M. Butler, "Seismic $Q-$ stratigraphy or dissipation," Geophysics 47, 16-24 (1982).

${ }^{25}$ W. H. Press, B. P. Flannery, S. A. Teukolsky, and W. T. Vetterling, $N u$ merical Recipes (Cambridge U.P., Cambridge, 1986), pp. 420-429.

${ }^{26}$ E. R. Kanasewich, Time Sequence Analysis in Geophysics (University of
Alberta, Edmonton, Canada, 1981), pp. 266-277.

${ }^{27}$ L. H. King, "Surficial geology of the Halifax-Sable Island map area," EMR Marine Sciences, Department of Energy, Mines and Resources, Canada, Paper 1, 16 pp. (1970).

${ }^{28}$ A. L. Anderson and L. D. Hampton, "A method for measuring in situ acoustic properties during sediment coring," in Physics of Sound in Marine Sediments, edited by L. Hampton (Plenum, New York, 1974).

${ }^{29}$ R. C. Courtney and L. A. Mayer, "Acoustic properties of fine grain sediments from Emerald Basin: Towards an inversion for physical properties using the Biot-Stoll model," J. Acoust. Soc. Am. (to be published).

${ }^{30}$ K. C. Baldwin, B. Celikkol, and A. J. Silva, "Marine sediment acoustic measurement system," Ocean Eng. 8, 489-496 (1981).

${ }^{31}$ R. D. Stoll, Sediment Acoustics (Springer-Verlag, New York, 1989).

${ }^{32}$ L. M. Brekhovskikh, Waves in Layered Media (Academic, New York, 1960).

${ }^{33}$ F. K. Levin, "Seismic velocities in transversely isotropic media, II," Geophysics 45, 3-17 (1980).

${ }^{34}$ E. L. Hamilton, "Elastic properties of marine sediments," J. Geophys. Res. 76, 579-604 (1971).

${ }^{35}$ R. S. Anderson, "Correlation of physical properties and velocity," in Ref. 28. 